\title{
Evaluation Methods Used to Assess mHealth Applications for Cardiovascular Disease: First Results of a Scoping Review
}

\author{
Felix HOLL ${ }^{\mathrm{a}, \mathrm{b}, 1}$, Jennifer KIRCHER ${ }^{\mathrm{a}}$ and Walter SWOBODA ${ }^{\mathrm{a}}$ \\ a DigiHealth Institute, Neu-Ulm University of Applied Sciences, Neu-Ulm, Germany \\ ${ }^{\mathrm{b}}$ Institute for Medical Information Processing, Biometry, and Epidemiology, Ludwig \\ Maximilian University of Munich, Munich, Geremany
}

\begin{abstract}
Potential benefits of mHealth applications are large for chronic diseases. To get an overview of how these applications are being evaluated, a scoping review is being conducted. First results show that single factors are most commonly assessed. The results of clinical outcome measures are the most common. Economic factors are the least common. A uniform framework that specifies different factors and metrics for the evaluation is not apparent.
\end{abstract}

Keywords. Mobile Health, Cardiovascular Diseases, Evaluation Methods

\section{Introduction}

The number of mHealth applications is growing fast. While their potential is large of chronic diseases, the evidence on the methods used to evaluate them is scarce [1]. The aim of this work is to provide an overview of the evaluation approaches used. Based on the results, a basis for an evaluation framework can be established, which will enable policy makers to assess the impact of mHealth applications using defined metrics.

\section{Methods}

A scoping review with two stages is being conducted to provide systematic evidence of how mHealth applications for cardiovascular diseases are evaluated in the published literature. The first step used an open literature search to get a preliminary overview and inform the development of the systematic search strategy as well as test the analysis plan. The second stage, is a systematic search with fixed search terms and inclusion and exclusion criteria. The results are loaded into the software Covidence and screened for inclusion and exclusion by two independent reviewers. Studies included in the analysis will be categorized by type of evaluation study, outcome metric and study population characteristics.

\footnotetext{
${ }^{1}$ Corresponding Author, Felix Holl, DigiHealth Institute Neu-Ulm University of Applied Sciences, Wileystr. 1, 89231 Neu-Ulm, Germany; E-mail: felix.holl@hnu.de
} 


\section{Results}

The first stage resulted in a list of inclusion and exclusion criteria and 28 studies met them and were analyzed and classified according to the "type of intervention" in four core areas. The "types of evaluation metrics" form a further variable for investigation. The most frequently examined parameters were "feasibility", "health outcome", "medication adherence", "self-care management", "acceptability" and "usability". The outcomes all relate to the end-user and their perspective. The least studied parameters were in the economic field. Currently, the authors are in the second stage of the review. 3859 publications were extracted for screening and are currently being screened and will then be analyzed. Results can be expected by the time of the conference.

\section{Discussion}

The first results showed various methods and instruments with which mobile health applications can be evaluated. Clinical outcome measures are the most common. Economic factors are the least common.

\section{Conclusion}

A uniform framework that specifies different factors and metrics for the evaluation is not apparent. It remains a challenge to select the right metrics and methods for evaluation as mHealth. The second stage will provide a formalized and more detailed analysis.

\section{References}

[1] World Health Organization, "mHealth: New horizons for health through mobile technologies," Observatory, vol. 3, no. June, pp. 66-71, 2011, doi: 10.4258/hir.2012.18.3.231. 